\title{
The Monte Carlo dynamics of a binary Lennard-Jones glass-forming mixture
}

\author{
L Berthier and W Kob \\ Laboratoire des Colloïdes, Verres et Nanomatériaux, UMR 5587, Université Montpellier II and \\ CNRS, 34095 Montpellier, France \\ E-mail: berthier@1cvn.univ-montp2.fr and kob@lcvn.univ-montp2.fr
}

Received 10 October 2006

Published 25 April 2007

Online at stacks.iop.org/JPhysCM/19/205130

\begin{abstract}
We use a standard Monte Carlo algorithm to study the slow dynamics of a binary Lennard-Jones glass-forming mixture at low temperature. We find that the Monte Carlo approach is by far the most efficient way to simulate a stochastic dynamics since the relaxation is about 10 times faster than in Brownian dynamics and about 30 times faster than in stochastic dynamics. Moreover, the average dynamical behaviour of the system is in quantitative agreement with that obtained using Newtonian dynamics, apart from at very short times where thermal vibrations are suppressed. We show, however, that dynamic fluctuations quantified by four-point dynamic susceptibilities do retain a dependence on the microscopic dynamics, as recently predicted theoretically.
\end{abstract}

\section{Introduction}

Numerical simulations play a major role among studies of the glass transition since, in contrast to experiments, the individual motion of a large number of particles can be followed at all times [1]. With present-day computers, it is possible to follow the dynamics of a simple glassforming liquid over more than eight decades of time, and over a temperature window in which average relaxation timescales increase by more than five decades. However, at the lowest temperatures studied, the relaxation is still orders of magnitude faster than in experiments performed close to the glass transition temperature. Nevertheless, it is now possible to numerically access temperatures which are low enough that many features associated to the glass transition physics can be observed: strong decoupling phenomena [2-4], clear deviations from fits to the mode-coupling theory [5] (which are experimentally known to hold only at high temperatures), and crossovers towards activated dynamics [6, 7].

Computer simulations usually study Newtonian dynamics (ND) by solving a discretized version of Newton's equations for a given pair interaction between particles [8]. Here, we study a glass-forming model in which a binary mixture of small and large particles interacts via a Lennard-Jones pair potential, a model introduced by Kob and Andersen (KA) [5]. It 
can also be interesting to study different types of microscopic dynamics for the same pair potential. If the dynamics satisfies detailed balance with respect to the Boltzmann distribution, all structural quantities remain unchanged, although the dynamics might be very different. In colloidal glasses, for instance, the particles undergo Brownian motion arising from collisions with the molecules of the solvent, and a stochastic dynamics is more appropriate. Theoretical considerations also suggest the study of different dynamics. Gleim et al studied a stochastic dynamics (SD) to investigate whether the relaxation of the KA binary mixture depended on its microscopic dynamics, their answer being 'no' [9]. In SD, a friction term and a random noise are added to Newton's equations, the amplitude of both terms being related by a fluctuationdissipation theorem. Szamel and Flenner recently used Brownian dynamics (BD) to study the same KA mixture [10]. In this description there are no momenta, and the positions evolve with a Langevin dynamics. They again find that relaxation using BD is very similar to that resulting from ND. They emphasize that even the deviations from mode-coupling fitting are similar in BD and ND, and conclude that momenta play no role in avoiding the mode-coupling singularity, contrary to previous claims [11], but in agreement with more recent ones [12].

Recently, it was also discovered that dynamic heterogeneity, that is, spatio-temporal fluctuations around the average dynamical behaviour, sensitively depends upon the microscopic dynamics $[6,7,13]$. In particular, a major role is played by conservation laws for energy and density. In the case of energy the mechanism can be physically understood as follows. For a rearrangement to take place in the liquid, the system has to locally cross an energy barrier. If the dynamics conserves the energy, particles involved in the rearrangement must borrow energy to the neighbouring particles. This 'cooperativity' might be unnecessary if energy can be locally supplied to the particles by an external heat bath. Conservation laws, therefore, might introduce dynamic correlations between particles, and dynamic fluctuations can be different when changing from Newtonian energy conserving dynamics to a stochastic thermostatted dynamics. This predicted influence of the microscopic dynamics on dynamic fluctuations [6, 7] was in fact our principal motivation for the present study.

In this article, we propose a third type of stochastic dynamics for the KA mixture and study in detail the dynamics of the system subjected to a standard Monte Carlo (MC) dynamics. We find that the MC approach is particularly efficient at relaxing the system since it is about 10 times faster than $\mathrm{BD}$ and 30 times faster than SD, while the average dynamics is still in quantitative agreement with ND. We are therefore in a position to study both the very lowtemperature average dynamics of the model and its dynamic fluctuations in detail, shedding new light on both aspects.

The paper is organized as follows. In section 2 we give details of the simulation technique and compare its efficiency to previously studied dynamics. In section 3 we present our numerical results. Section 4 concludes the paper.

\section{An efficient simulation technique}

We study a binary Lennard-Jones mixture made of $N_{A}=800$ and $N_{\mathrm{B}}=200$ particles of types $A$ and $B$, respectively. Particles interact with the following Lennard-Jones pair potential:

$$
\phi_{\alpha \beta}^{\mathrm{LJ}}(r)=4 \epsilon_{\alpha \beta}\left[\left(\frac{\sigma_{\alpha \beta}}{r}\right)^{12}-\left(\frac{\sigma_{\alpha \beta}}{r}\right)^{6}\right],
$$

where $\alpha, \beta \in[A, B]$ and $r$ is the distance between the interacting pair of particles. Interaction parameters $\epsilon_{\alpha \beta}$ and $\sigma_{\alpha \beta}$ are chosen to prevent crystallization and can be found in [5]. The length and energy are given in the standard Lennard-Jones units $\sigma_{A A}$ (particle diameter), and $\epsilon_{A A}$ (interaction energy), where the subscript $A$ refers to the majority species. The potential is truncated and shifted at a distance $r=2.5$. Previous work $[1,5]$ has shown that the dynamics 


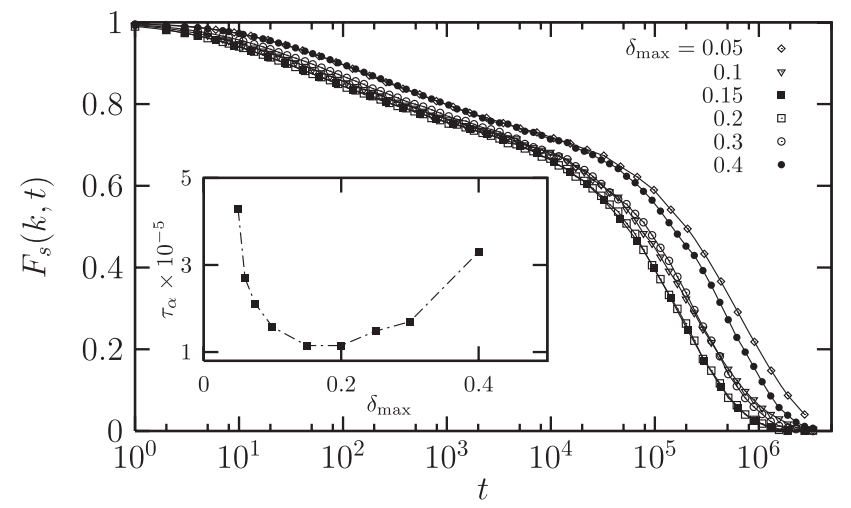

Figure 1. Self-intermediate scattering function, equation (2), at $T=0.5$ and $k=7.21$ for various values of $\delta_{\max }$. Inset: the evolution of the relaxation time with $\delta_{\max }$ unambiguously defines an optimal value $\delta_{\max } \approx 0.15$ for our simulations.

becomes slow below $T \approx 1.0$, while the fitted mode-coupling temperature for this system is $T_{\mathrm{c}} \approx 0.435$, although deviations from mode-coupling behaviour become noticeable already below $T \approx 0.47$.

We have implemented a standard Monte Carlo dynamics [8] for the pair potential in equation (1). An elementary move can be described as follows. A particle, $i$, located at the position $\mathbf{r}_{i}$, is chosen at random. The energy cost, $\Delta E_{i}$, to move particle $i$ from position $\mathbf{r}_{i}$ to a new position $\mathbf{r}_{i}+\delta \mathbf{r}$ is evaluated, $\delta \mathbf{r}$ being a random vector comprised in a cube of linear length $\delta_{\max }$ centred around the origin. The Metropolis acceptance rate, $p=\min \left(1, \mathrm{e}^{-\beta \Delta E_{i}}\right)$, where $\beta=1 / T$ is the inverse temperature, is then used to decide whether the move is accepted. In the following, one Monte Carlo timestep represents $N=N_{A}+N_{\mathrm{B}}$ attempts to make such an elementary move, and timescales are reported in this unit.

The one degree of freedom that remains to be fixed is $\delta_{\max }$, which determines the average lengthscale of elementary moves. If chosen too small, the energy costs are very small, and most of the moves are accepted, but the dynamics is very slow because it takes a long time for particles to explore their cage. On the other hand, too large displacements will on average be very costly in energy, and acceptance rates can become prohibitively small. We seek a compromise between these two extremes by monitoring the dynamics at a moderately low temperature, $T=0.5$, for several values of $\delta_{\max }$. As the most sensitive indicator of the relaxational behaviour, we measure the contribution from the majority species $A$ to the selfintermediate scattering function,

$$
F_{\mathrm{s}}(\mathbf{k}, t)=\left\langle\frac{1}{N_{A}} \sum_{j=1}^{N_{A}} \mathrm{e}^{\mathrm{i} \mathbf{k} \cdot\left[\mathbf{r}_{j}(t)-\mathbf{r}_{j}(0)\right]}\right\rangle .
$$

We spherically average over wavevectors of comparable magnitude, and present results for $|\mathbf{k}|=7.21$, which corresponds to the first diffraction peak in the static structure factor of the liquid. In figure 1 , we present our results for $\delta_{\max }$ values between 0.05 and 0.4 . As expected, we find that the relaxation is slow both at small and large values of $\delta_{\max }$, and is most efficient for intermediate values. Interestingly, we also note that the overall shape of the self-intermediate scattering function does not sensitively depend on $\delta_{\max }$.

We define a typical relaxation time as $F_{\mathrm{s}}\left(k, \tau_{\alpha}\right)=\mathrm{e}^{-1}$ and show its $\delta_{\max }$ dependence in the inset of figure 1 . A clear minimum is observed at the optimal value of $\delta_{\max } \approx 0.15$. In the rest of the paper we only present data obtained for this value. 

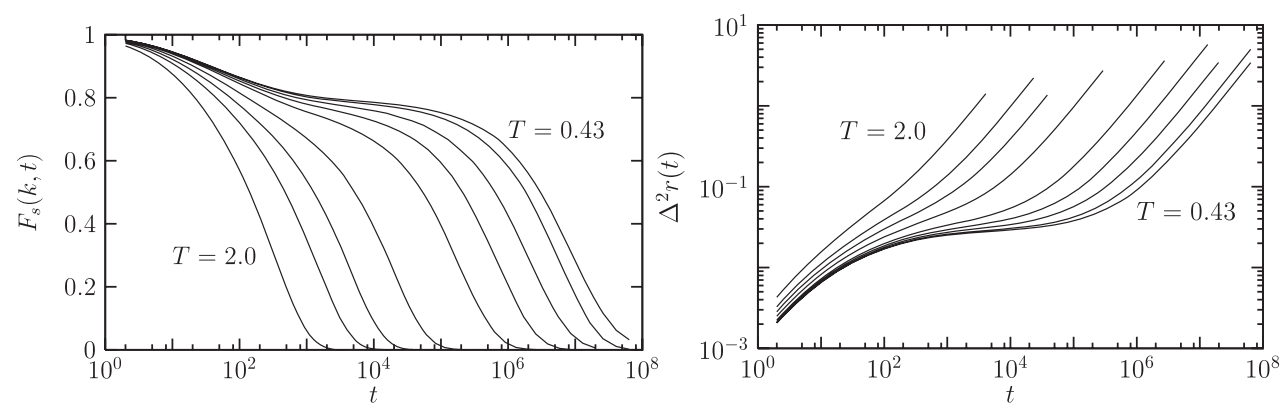

Figure 2. Left: self-intermediate scattering function, equation (2), for $k=7.21$ and temperatures $T=2.0,1.0,0.75,0.6,0.5,0.47,0.45,0.435$, and 0.43 (from left to right). Right: mean-squared displacement, equation (3), for the same temperatures in the same order.

As compared to previously studied dynamics, we find that, when expressed in numbers of integration timesteps, structural relaxation in Monte Carlo simulations is marginally faster than in Newtonian dynamics, but 30 times faster than in stochastic dynamics [9], and 10 times faster than in Brownian dynamics [10]. We conclude therefore that the MC approach is by far the most efficient way to perform stochastic molecular simulations of the present glass-forming material.

The relative inefficiency of both $\mathrm{BD}$ and $\mathrm{SD}$ is due to the stochastic nature of their microscopic equations of motion. It is well known that small integration timesteps are required for accurate integration of stochastic equations of motion, in particular to maintain the delicate balance between friction and noise required for the system to converge towards the correct equilibrium distribution [8]. No such constraint exists for MC dynamics, where elementary moves can be made arbitrarily large. Equilibrium only requires detailed balance to be fulfilled, and this is always the case with the Metropolis algorithm described above. With larger elementary moves, particles can efficiently explore their cage and relaxation is much faster. This physical interpretation is also supported by the optimal value $\delta_{\max }=0.15$ that we report, which corresponds to a mean-squared displacement of 0.225 , very close to the plateau observed in the mean-squared displacement shown in figure 2, which can be taken as a rough estimate of the cage size. Monte Carlo simulations can of course be made even more efficient by implementing for instance swaps between particles, or using parallel tempering. The dynamical behaviour, however, is then strongly affected by such non-physical moves and only equilibrium thermodynamics can be studied. Since we want to conserve a physically realistic dynamics, we cannot use such improved schemes.

We have performed simulations at temperatures between $T=2.0$ and $T=0.43$, the latter being smaller than the fitted mode-coupling temperature. For each temperature we have simulated ten independent samples to improve the statistics. Initial configurations were taken as the final configurations obtained from previous work performed with ND [6, 7], so that production runs could be started immediately. For each sample, production runs lasted at least $15 \tau_{\alpha}$ (at $T=0.43$ ), and much longer for higher temperatures.

\section{Results}

\subsection{Average dynamics}

The self-intermediate scattering function, equation (2), is shown in figure 2 for temperatures decreasing from $T=2.0$ down to $T=0.43$. These curves present well-known features. The 
dynamics at high temperature is fast and has an exponential nature. When the temperature is decreased below $T \approx 1.0$, a two-step decay, the slower being strongly non-exponential, becomes apparent. Upon decreasing the temperature further, the slow process dramatically slows down by about five decades, while clearly conserving an almost temperature-independent non-exponential shape, as already reported for ND [5].

Finally, as reported for SD [9], we also find that the first process, the decay towards a plateau, slows down considerably when decreasing the temperature. This process, called 'critical decay' in the language of mode-coupling theory [14], is not observed when using ND, because it is obscured by the thermal vibrations occurring at high frequencies. Although the plateau seen in $F_{\mathrm{s}}(k, t)$ is commonly interpreted as 'vibrations of a particle within a cage', the data in figure 2 discard this view. From direct visualization of the particles' individual dynamics it is obvious that vibrations take place in just a few MC timesteps, while the decay towards the plateau can be as long as $10^{4}$ time units at the lowest temperatures studied here. This decay is therefore necessarily more complex, and most probably cooperative in nature. This interpretation is supported by recent theoretical studies where a plateau is observed in two-time correlators of lattice models where local vibrations are indeed completely absent [15]. A detailed atomistic description of this process has not yet been reported, but would indeed be very interesting.

Next, we study the mean-squared displacement for the majority specie. It is defined as

$$
\Delta^{2} r(t)=\frac{1}{N_{A}} \sum_{i=1}^{N_{A}}\left\langle\left|\mathbf{r}_{i}(t)-\mathbf{r}_{i}(0)\right|^{2}\right\rangle,
$$

and we present its temperature evolution in figure 2, which mirrors the evolution of the self-intermediate scattering function in the same figure. Since we are studying a stochastic dynamics, displacements are diffusive at both short and long timescales. The plateau observed in $F_{\mathrm{s}}(k, t)$ now translates into a sub-diffusive regime in the mean-squared displacements separating the two diffusive regimes. At the lowest temperature studied, when $t$ changes by three decades from $2 \times 10^{2}$ to $2 \times 10^{5}$, the mean-squared displacement changes by a mere factor of 2.2, from 0.02 to 0.044 . Particles are therefore nearly arrested for several decades of times, before eventually entering the diffusing regime which allows for the relaxation of the structure of the liquid.

\subsection{Comparison to Newtonian and stochastic dynamics}

The previous subsection has shown that the Monte Carlo dynamics of the KA mixture is qualitatively similar to that reported for ND, apart from at relatively short times where the effect of thermal vibrations is efficiently suppressed. We now compare our results more quantitatively with the dynamical behaviour observed using ND.

In figure 3 we compare the time dependence of the self-intermediate scattering function for three types of dynamics: the present Monte Carlo data, the Newtonian dynamics data taken from [6], and the stochastic dynamics results from [9], all obtained for the same parameters, $k=7.21$ and $T=0.45$. We have rescaled the time to obtain maximum overlap in the longtime relaxation of the three curves. Quite strikingly, the SD and MC data perfectly overlap over the complete time range (eight decades of time) of the simulation. Indeed the SD dotted line is barely visible below the full line of the MC data in figure 3. This confirms our claim that the MC approach defines a physically relevant microscopic dynamics, since it is completely equivalent to SD with the major advantage that it is 30 times faster, at least for the KA mixture.

In figure 3 , we also confirm that the approach to the plateau is different in MC/SD and ND. In the latter, phonon-like vibrations affect the initial decay of $F_{\mathrm{s}}(k, t)$. For instance, a 


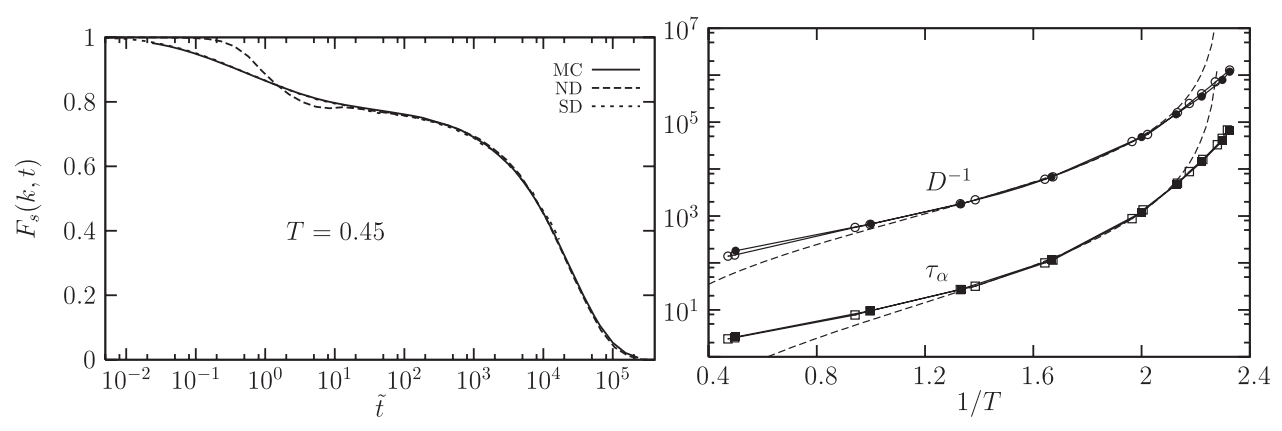

Figure 3. Left: comparison of the self-intermediate scattering function for $k=7.21$, and $T=0.45$, obtained in Monte Carlo (MC) dynamics in this work, Newtonian dynamics (ND) in [6], and stochastic dynamics (SD) in [9]. Time is rescaled to obtain maximum overlap at large times. MC and SD agree over the complete time range (indeed the SD dotted line is barely visible below the full MC line), while MC and ND only agree when $F_{\mathrm{S}}(k, t)$ is close to the plateau and below. The dip observed at short time in ND is due to thermal vibrations suppressed in both SD and MC. Right: temperature evolution of the alpha-relaxation time $\tau_{\alpha}(T)$ and the inverse of the self-diffusion constant $1 / D(T)$ in an Arrhenius plot. Open symbols are for ND, closed symbols for MC (vertically shifted to obtain maximum overlap with ND data), and the dashed lines are power-law fits to a divergence at $T_{\mathrm{c}}=0.435$, as originally reported in [5].

shallow dip, generally attributed to the 'Boson peak', is observed at low temperature in ND; see the dashed line in figure 3. The long-time decay of the self-intermediate scattering function, however, is in full quantitative agreement for the three dynamics. This agreement was the main claim of [9], extended to BD in [10] and for the MC approach in the present work.

Since all dynamics display similar long-time relaxation, it is sensible also to quantitatively compare the temperature evolution of the relaxation times, $\tau_{\alpha}(T)$, already defined above. This is done in figure 3, where we use a standard representation in which an Arrhenius slowing down over a constant energy barrier, $\tau_{\alpha} \sim \exp (E / T)$, would appear as a straight line. The data clearly show some upwards bending in figure 3, a super-Arrhenius behaviour typical of fragile glass-formers. We find that the temperature evolution of the alpha-relaxation time measured in MC simulations is in complete quantitative agreement with that obtained from ND, over the complete temperature range $T=2.0 \rightarrow 0.43$. In particular the quality of a power-law fit of the slowing down, $\tau_{\alpha} \sim\left(T-T_{\mathrm{c}}\right)^{-\gamma}$, as suggested by mode-coupling theory, is similar for both dynamics [5,9]. We have shown such a fit through our data, using the value $T_{\mathrm{c}}=0.435$ determined in [5]. The fit describes the data over about 2.5 decades. Deviations from the mode-coupling fit appear below $T \approx 0.47$, and become obvious when $T$ is decreased further.

In figure 3 we also show the temperature evolution of the self-diffusion constant, defined from the long-time limit of the mean-square displacement as

$$
D=\lim _{t \rightarrow \infty} \frac{\Delta^{2} r(t)}{6 t}
$$

The behaviour of the diffusion constant is qualitatively very close to that of the alpha-relaxation time, and all the above remarks apply. The well-known difference between the two quantities is a slightly stronger temperature evolution of $\tau_{\alpha}$, implying a well-studied decoupling between translational diffusion and structural relaxation in this system [1,4], which is therefore very similar for different types of dynamics.

Theoretically, an identical relaxation within MC/SD/BD/ND is an important prediction of mode-coupling theory [14] because the theory uniquely predicts the dynamical behaviour 


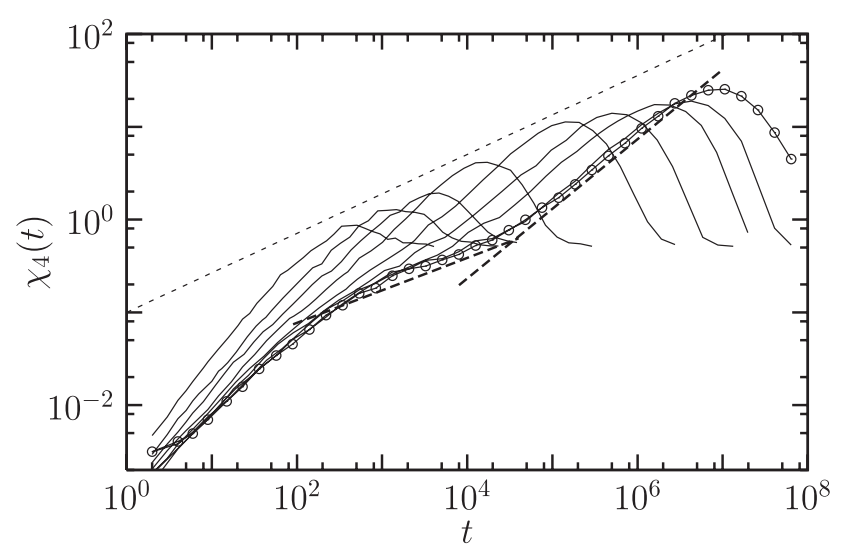

Figure 4. Four-point susceptibility, equation (5), for the same temperatures as in figure 2, decreasing from left to right. We have highlighted with open circles the data at $T=0.43$, which are fitted with two power laws shown as dashed lines with exponents 0.35 and 0.75 at short and large times, respectively.

from static density fluctuations. Gleim et al argue that their finding of a quantitative agreement between SD and ND is a nice confirmation of this non-trivial mode-coupling prediction [9]. Szamel and Flenner [10] confirmed this claim using BD, and argued further that even deviations from mode-coupling predictions are identical. We confirm the validity of this statement even below $T_{\mathrm{c}}$, showing that the agreement between different dynamics, although indeed predicted by mode-coupling theory, is certainly valid at a much more general level. Similarly to Szamel and Flenner, we note that deviations from a power-law divergence cannot be attributed to coupling to currents which are expressed in terms of particle velocities. In our MC simulations we have no velocities, so that avoiding the mode-coupling singularity is not due to the hydrodynamic effects pointed out in [11] (see [12] for more recent theoretical viewpoints).

\subsection{Multi-point susceptibility}

Having established the ability of MC simulations to efficiently reproduce the average slow dynamics obtained from ND simulations, we now turn to the study of the dynamic fluctuations around the average dynamical behaviour, i.e. to dynamic heterogeneity.

Dynamic fluctuations can be studied through the four-point susceptibility, $\chi_{4}(t)$, which quantifies the strength of the spontaneous fluctuations around the average dynamics by their variance,

$$
\chi_{4}(t)=N_{A}\left[\left\langle f_{\mathrm{s}}^{2}(\mathbf{k}, t)\right\rangle-F_{\mathrm{s}}^{2}(\mathbf{k}, t)\right],
$$

where $f_{\mathrm{s}}(\mathbf{k}, t)=N_{A}^{-1} \sum_{j} \cos \left(\mathbf{k} \cdot\left[\mathbf{r}_{j}(t)-\mathbf{r}_{j}(0)\right]\right)$ represents the real part of the instantaneous value of the self-intermediate scattering function, so that $F_{\mathrm{s}}(\mathbf{k}, t)=\left\langle f_{\mathrm{s}}(\mathbf{k}, t)\right\rangle$. As shown by equation (5), it is clear that $\chi_{4}(t)$ will be large if run-to-run fluctuations of the self-intermediate scattering functions are large. This is the case when the local dynamics becomes spatially correlated, as already discussed in several papers [16-21].

We show the time dependence of the dynamic susceptibility $\chi_{4}(t)$ obtained from our MC simulations for various temperatures in figure 4. As predicted theoretically in [20], we find that $\chi_{4}(t)$ presents a complex time evolution, closely related to the time evolution of the self-intermediate scattering function. Overall, $\chi_{4}(t)$ is small at both small and large times when dynamic fluctuations are small. There is therefore a clear maximum observed for times 


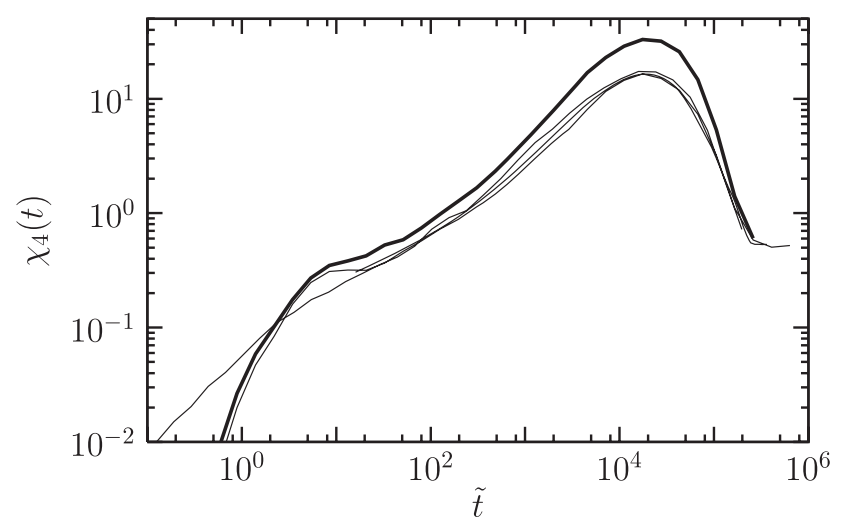

Figure 5. Four-point susceptibility for various dynamics and ensembles at $T=0.45$. As in figure 3 , times have been rescaled to obtain the maximum overlap in the long-time regime. The three overlapping thin lines represent data for ND- $N V E$ dynamics, SD, and MC, while the thick line represents ND- $N V T$ data, for which dynamic fluctuations are clearly larger, as predicted theoretically and discussed in [6].

comparable to $\tau_{\alpha}$, where fluctuations are most prominent. The position of the maximum then shifts to larger times when the temperature is decreased, tracking the alpha-relaxation. The most important physical information revealed by these curves is the fact that the amplitude of the peak grows when the temperature decreases. This is direct evidence that spatial correlations grow when the glass transition is approached.

The two-step decay of the self-intermediate scattering function translates into a two-powerlaw regime for $\chi_{4}(t)$ approaching its maximum. We have fitted these power laws, $\chi_{4}(t) \sim t^{a}$, followed by $\chi_{4}(t) \sim t^{b}$, with the exponents $a=0.35$ and $b=0.75$, in figure 4 . We have intentionally used the notation $a$ and $b$ for these exponents which are predicted, within modecoupling theory, to be equal to the standard exponents also describing the time dependence of intermediate scattering functions [14]. Our findings are in good agreement with previously reported values for $a$ and $b$. See [7, 20] for a more extensive discussion and comparison to other theoretical predictions.

We finally compare the dynamic susceptibility for various dynamics. In figure 5, we present the time evolution of $\chi_{4}(t)$ for a given temperature, $T=0.45$ and four different dynamics: the present MC data, data from SD obtained in [6], data for ND in the microcanonical $(N V E)$ ensemble from [6], and data for ND in the canonical $(N V T)$ ensemble from [6]. To perform this comparison, we have again rescaled times to obtain the maximum overlap in the long-time region. In figure 5 it is obvious that three curves are identical: ND$N V E, \mathrm{MC}$ and SD data perfectly overlap near the maximum of $\chi_{4}(t)$ and have similar time dependences, apart from at very short times. On the other hand, the ND- $N V T$ data display a different time dependence and reveal considerably larger dynamic fluctuations in the long-time regime.

We conclude therefore that, contrary to the average dynamics, the dynamic fluctuations quantified through the four-point susceptibility do retain a dependence upon the microscopic dynamics since canonical estimates of $\chi_{4}(t)$ are different for ND and for MC/SD/BD. Although perhaps counterintuitive at first sight, we find that dynamics with a stochastic heat-bath display dynamic fluctuations similar to those measured using microcanonical ND, while fluctuations are much larger in canonical ND simulations. As mentioned in the introduction, this confirms the idea that the energy conservation (implied by Newton's equations of motion) might lead to 
an amplification of dynamic fluctuations. With hindsight, this is not such a surprising result: the specific heat, after all, also behaves differently in different statistical ensembles. The ensemble dependence and dependence upon the microscopic dynamics are the main subjects of two recent papers $[6,7]$.

There is an experimentally relevant consequence of these findings. The difference between the microcanonical and canonical values of the dynamic fluctuations in ND can be shown to be equal to [13]

$$
\chi_{4}^{N V T}(t)-\chi_{4}^{N V E}(t)=\frac{T^{2}}{c_{V}}\left(\frac{\partial F_{\mathrm{s}}(\mathbf{k}, t)}{\partial T}\right)^{2},
$$

where $c_{V}$ is the constant volume specific heat expressed in $k_{\mathrm{B}}$ units. As shown in figure 5, the temperature derivative in equation (6) represents in fact the major contribution to $\chi_{4}^{N V T}$, meaning that the term $\chi_{4}^{N V E}$ can be neglected in equation (6). Since the right-hand side of (6) is more easily accessible in an experiment than $\chi_{4}$ itself, equation (6) opens the possibility of an experimental estimate of the four-point susceptibility. This finding, and its experimental application to supercooled glycerol and hard sphere colloids, constitute the central results of [13].

\section{Conclusion}

We have implemented a standard Monte Carlo dynamics on the well-known binary LennardJones mixture introduced by KA. We have shown that the resulting average dynamics is in full quantitative agreement with results from Newtonian dynamics, while being considerably faster than previously studied stochastic dynamics, namely Brownian and stochastic dynamics. We have therefore at our disposal an efficient numerical technique to simulate the stochastic dynamics of the KA mixture at low temperature. This allowed us to show, in particular, that dynamic fluctuations retain a dependence upon the microscopic dynamics since four-point dynamical susceptibilities evaluated in the canonical ensemble for ND and MC quantitatively differ, because the energy conservation of Newton's equations amplify dynamic fluctuations.

\section{Acknowledgments}

We wish to thank J L Barrat for useful discussions, and G Biroli, J P Bouchaud, K Miyazaki, and D Reichman for our recent collaboration $[6,7]$, which initially motivated this work.

\section{References}

[1] Andersen H C 2005 Proc. Natl Acad. Sci. 1026686

[2] Perera D N and Harrowell P 1999 J. Chem. Phys. 1115441

[3] Yamamoto R and Onuki A 1998 Phys. Rev. Lett. 814915

[4] Berthier L 2004 Phys. Rev. E 69020201

[5] Kob W and Andersen H C 1994 Phys. Rev. Lett. 731376

Kob W and Andersen H C 1995 Phys. Rev. E 534134

Kob W and Andersen H C 1995 Phys. Rev. E 514626

[6] Berthier L, Biroli G, Bouchaud J-P, Kob W, Miyazaki K and Reichman D R 2006 Preprint cond-mat/0609656

[7] Berthier L, Biroli G, Bouchaud J-P, Kob W, Miyazaki K and Reichman D R 2006 Preprint cond-mat/0609658

[8] Allen M and Tildesley D 1987 Computer Simulation of Liquids (Oxford: Oxford University Press)

[9] Gleim T, Kob W and Binder K 1998 Phys. Rev. Lett. 81004404

[10] Szamel G and Flenner E 2004 Europhys. Lett. 67779

[11] Das S P and Mazenko G F 1986 Phys. Rev. A 342265

[12] Andreanov A, Biroli G and Lefèvre A 2006 J. Stat. Mech. P07008 
Cates M E and Ramaswamy S 2005 Phys. Rev. Lett. 96135701

[13] Berthier L, Biroli G, Bouchaud J-P, Cipelletti L, El Masri D, L’Hôte D, Ladieu F and Pierno M 2005 Science 3101797

[14] Götze W 1999 J. Phys.: Condens. Matter 11 A1

[15] Sellitto M, Biroli G and Toninelli C 2005 Europhys. Lett. 69496

[16] Franz S and Parisi G 2000 J. Phys.: Condens. Matter 126335

[17] Franz S, Donati C, Parisi G and Glotzer S C 1999 Phil. Mag. B 791827

[18] Bennemann C, Donati C, Baschnagel J and Glotzer S C 1999 Nature 399246

[19] Lačević N, Starr F W, Schroder T B and Glotzer S C 2003 J. Chem. Phys. 1197372

[20] Toninelli C, Wyart M, Biroli G, Berthier L and Bouchaud J-P 2005 Phys. Rev. E 71041505

[21] Mayer P, Bissig H, Berthier L, Cipelletti L, Garrahan J P, Sollich P and Trappe V 2004 Phys. Rev. Lett. 93115701 